\title{
Long-Time Behavior of Solutions for a Class of Nonlinear Higher Order Kirchhoff Equation
}

\author{
Guoguang Lin*, Ying Jin \\ School of Mathematics and Statistics, Yunnan University, Kunming, China \\ Email address: \\ gglin@ynu.edu.cn (Guoguang Lin), 15758542844@163.com (Ying Jin) \\ ${ }^{*}$ Corresponding author
}

\section{To cite this article:}

Guoguang Lin, Ying Jin. Long-Time Behavior of Solutions for a Class of Nonlinear Higher Order Kirchhoff Equation. American Journal of Applied Mathematics. Vol. 7, No. 1, 2019, pp. 21-29. doi: 10.11648/j.ajam.20190701.14

Received: April 1, 2019; Accepted: May 10, 2019; Published: June 3, 2019

\begin{abstract}
In this paper, we study a class of the long-time behavior of solutions to initial-boundary value problems for higher order equations with nonlinear source term and strong damping term. First of all, give some space and marks as well as the basic assumption of stress and nonlinear source term, take the inner product on both sides of the equation and obtain a priori estimate of the global smooth solution of the equation by using Holder inequality, Yong inequality, Poincare inequality and Gronwall inequality. Then prove the existence of the global solution of the equation by using the Galerkin finite element method. The uniqueness of the global solution of the equation is proved, and then the bounded absorption set of the solution semi-group is constructed by a priori estimate. It is proved that the solution semi-group is uniformly bounded and completely continuous in the interior, thus the global attractor family of the equation is obtained. Then the original equation is linearized, and the differentiability of the solution semi-group is proved, and the line is further proved. The decay of the volume element of the sexualization problem is studied, and the finite Hausdorff dimension and Fractal dimension of the global attractor family are obtained.
\end{abstract}

Keywords: Kirchhoff Equation, The Existence and Uniqueness of Solutions, Global Attractor Family, Hausdorff Dimension, Fractal Dimension

\section{Introduction}

In this paper, we mainly study the higher order Kirchhoff equation with strong nonlinear damping term

$$
\begin{array}{r}
u_{t t}+M\left(\left\|D^{m} u\right\|^{2}\right)(-\Delta)^{m} u+\beta(-\Delta)^{m} u_{t}+g\left(\|\left. u\right|^{2}\right) u=f(x) \\
u(x, t)=0, \frac{\partial^{i} u}{\partial v^{i}}=0, i=1,2, \cdots, m-1, x \in \partial \Omega, t>0 \\
u(x, 0)=u_{0}(x), u_{t}(x, 0)=u_{1}(x), x \in \Omega \subset R^{n}
\end{array}
$$

where $m>1, \Omega$ is a bounded region on $R^{n}$ with smooth Dirichlet boundary $\partial \Omega$ and $g\left(|u|^{2}\right) u$ is a non-linear source term.

Since Kirchhoff[1] proposed the Kirchhoff rope model for studying the free vibration of elastic strings in 1883, many scholars have devoted themselves to the study of Kirchhoff equation. In 1997, Kosuke Ono [2] studied the initial-boundary value problem of the nonlinear Kirchhoff wave equation with strong dissipation term

$$
\begin{gathered}
u_{t t}=\|D u\|^{2 \gamma} \Delta u-\Delta_{u_{t}}=|u|^{\alpha} u \\
u(0)=u_{0}, u_{t}(0)=u_{1} \\
\left.u\right|_{\partial \Omega}=0
\end{gathered}
$$

by using the modified potential well method and concave method, it is proved that when the initial energy $E\left(u_{0}, u_{1}\right)=\left\|u_{1}\right\|^{2}+\frac{1}{\gamma+1}\left\|D u_{0}\right\|^{2(\gamma+1)}-\frac{2}{\alpha+2}\left\|u_{0}\right\|_{\alpha+2}^{\alpha+2}$ related to the equation is non-negative and small enough, a unique (weak) solution exists globally in time and has a certain attenuation characteristic; when the initial energy $E\left(u_{0}, u_{1}\right)$ is 
negative, the solution will explode in a certain time.

In 2007, Xiaoming Fan and Yaguang Wang [3] studied the low-order stochastic wave equation with nonlinear damping and white noise existence and fractal dimension of compact random attractors.

$$
u_{t t}+\alpha u_{t}+h(u) u_{t}-\Delta u+f(u, t)-g(x, t)=q(x) \dot{W}
$$

In 2010, Zhijian Yang and Yunqing Wang [4] studied the long-term behavior of solutions of low-order Kirchhoff equation with strong damping term

$$
\begin{gathered}
u_{t t}-M\left(\|D u\|^{2}\right) \Delta u-\Delta u_{t}+h\left(u_{t}\right)+g(u)=f(x), \\
\left.u\right|_{\partial \Omega}=0, \\
u(x, 0)=u_{0}(x), u_{t}(x, 0)=u_{1}(x),
\end{gathered}
$$

where $M(s)=1+s^{\frac{m}{2}}, 1 \leq m \leq \frac{4}{(N-2)^{+}}$, when $N=1$, the equation does not contain dissipation term $-\Delta u_{t}$ to describe the small vibration of elastic strings. On this basis, in 2011, Zhijian Yang and Jianling Cheng [5] once again studied the long-term behavior of solutions of low-order Kirchhoff equation with strong damping:

$$
\begin{gathered}
u_{t t}-M\left(\|D u\|^{2}\right) \Delta u-\Delta u_{t}+h\left(u_{t}\right)+g(x, u)=f(x), \\
\left.u\right|_{\partial \Omega}=0 \\
u(x, 0)=u_{0}(x), u_{t}(x, 0)=u_{1}(x)
\end{gathered}
$$

where $M(s)=1+s^{\frac{m}{2}}, m \geq 1$. For more information on Kirchhoff equation, please refer to references [6-20].

On the basis of the lower-order Kirchhoff-type equation studied by previous scholars, this paper studies the global attractor family and its finite dimension estimation for the higher-order Kirchhoff-type equation with strong non-linear damping terms. The structure of this paper is as follows: The first part is mainly about some basic assumptions; the second part is to prove the existence and uniqueness of the global solution by using prior estimation and Galerkin method, and prove that the global solution is unique. In the third part, we first prove the differentiability of solution semi-groups, and then estimate the finite Hausdorff dimension and Fractal dimension of global attractor family.

\section{Basic Assumptions}

For narrative convenience, define the following spaces and marks:

$$
\nabla=D \quad, \quad H=L^{2}(\Omega) \quad, \quad H_{0}^{m}(\Omega)=H^{m}(\Omega) \cap H_{0}^{1}(\Omega) \quad \text {, }
$$
$H_{0}^{m+k}(\Omega)=H^{m+k}(\Omega) \cap H_{0}^{1}(\Omega), C, C_{i}(i=1,2, \cdots, 21)$ is a constant greater than $0, E_{k}=H_{0}^{m+k}(\Omega) \times H_{0}^{k}(\Omega), \quad(k=0,1,2 \cdots m)$, when $k=0, E_{0}=H_{0}^{m} \times L^{2}(\Omega)$.

Definitions (.,.) and $\|\cdot\|=\|\cdot\|_{L^{2}}$ represent the inner product and norm of $H$, respectively

$$
(u, v)=\int_{\Omega} u(x) v(x) d x,(u, u)=\|u\|^{2} .
$$

Let $A_{k}$ be a weak global attractor family from $E_{0}$ to $E_{k}$, and $B_{0 k}$ be a bounded absorption set from $E_{0}$ to $E_{k}$, where $k=1,2 \cdots, m$.

Kirchhoff stress term $M(s)$ satisfies the following condition:

(A) $M(s) \in C^{2}(\Omega)$;

$$
1<\mu_{0} \leq M(s) \leq \mu_{1}, \mu=\left\{\begin{array}{ll}
\mu_{0}, & \frac{d}{d t}\left\|D^{m+k} u\right\|^{2} \geq 0 \\
\mu_{1}, & \frac{d}{d t}\left\|D^{m+k} u\right\|<0
\end{array} .\right.
$$

Nonlinear term $g\left(|u|^{2}\right) u$ satisfies the following condition:

(B) Let $g\left(|u|^{2}\right) u$ be locally bounded and measurable, $g\left(|u|^{2}\right) \in C^{2}(\Omega)$.

\section{Existence of Global Attractor Family}

In this part, we first estimate the solution of problem (1) - (3) a priori, then prove the existence and uniqueness of global solution by Galerkin method. Finally, problem (1) - (3) generates solution semi-groups and obtains global attractor family by the properties of solution semi-groups.

Lemma 1 Assuming that the Kirchhoff stress term $M(s)$ and the non-linear term $g\left(|u|^{2}\right) u$ satisfy the conditions $(A)$ and $(B), f(x) \in H \quad, \quad\left(u_{0}, u_{1}\right) \in E_{k} \quad(k=0,1,2, \cdots, m)$, $v=u_{t}+\varepsilon a, \mathcal{E}>0$, respectively, the smooth solution $(u, v) \in E_{k}$ of the initial-boundary value problem (1) - (3) satisfies the following inequality

$$
\|(u, v)\|_{E_{k}}^{2}=\left\|D^{m+k} u\right\|^{2}+\left\|D^{k} v\right\|^{2} \leq \phi(0) e^{-\varkappa}+C\left(1-e^{-\chi}\right),
$$

therefore, there exists a non-negative real number $C\left(R_{1}\right)$ and $t=t_{2}(\Omega)>0$,

$$
\gamma=\min \left\{\frac{\beta \lambda_{1}^{m}}{2}-2 \varepsilon-\varepsilon^{2}, \frac{2 \varepsilon \mu_{0}-\varepsilon^{2} \lambda_{1}^{-m}-\beta \varepsilon^{2}-C_{1} \lambda_{1}^{-m}}{\mu}, 2 \varepsilon\right\},
$$

such that

$$
\varlimsup_{t \rightarrow \infty}\|(u, v)\|_{E_{k}}^{2} \leq \frac{C}{\gamma}=C\left(R_{1}\right) \quad\left(t>t_{2}\right)
$$

Proof By taking the inner product of 
$(-\Delta)^{k} v=(-\Delta)^{k} u_{t}+(-\Delta)^{k} \varepsilon u$ and equation (1), we can get

$$
\left(u_{t t}+M\left(\left\|D^{m} u\right\|^{2}\right)(-\Delta)^{m} u+\beta(-\Delta)^{m} u_{t}+g\left(|u|^{2}\right) u,(-\Delta)^{k} v\right)=\left(f(x),(-\Delta)^{k} v\right)
$$

The items in successive processing (4) are as follows:

$$
\left(u_{t t},(-\Delta)^{k} v\right)=\frac{1}{2} \frac{d}{d t}\left\|D^{k} v\right\|^{2}-\varepsilon\left\|D^{k} v\right\|^{2}+\varepsilon^{2}\left(D^{k} u, D^{k} v\right) \geq \frac{1}{2} \frac{d}{d t}\left\|D^{k} v\right\|^{2}-\frac{2 \varepsilon+\varepsilon^{2}}{2}\left\|D^{k} v\right\|^{2}-\frac{\varepsilon^{2} \lambda_{1}^{-m}}{2}\left\|D^{m+k} u\right\|^{2}
$$

according to hypothesis $(A)$, we can get

$$
\left(M\left(\left\|D^{m} u\right\|^{2}\right)(-\Delta)^{m} u,(-\Delta)^{k} v\right)=M\left(\left\|D^{m} u\right\|^{2}\right) \frac{1}{2} \frac{d}{d t}\left\|D^{m+k} u\right\|^{2}+\varepsilon M\left(\left\|D^{m} u\right\|^{2}\right)\left\|D^{m+k} u\right\|^{2} \geq \frac{1}{2} \mu \frac{d}{d t}\left\|D^{m+k} u\right\|^{2}+\varepsilon \mu_{0}\left\|D^{m+k} u\right\|^{2}
$$

according to Holder inequality, Young inequality and Poincare inequality, there are

$$
\left(\beta(-\Delta)^{m} u_{t},(-\Delta)^{k} v\right)=\beta\left\|D^{m+k} v\right\|^{2}-\beta \varepsilon\left(D^{m+k} u, D^{m+k} v\right) \geq \frac{\beta \lambda_{1}^{m}}{4}\left\|D^{k} v\right\|^{2}+\frac{\beta \lambda_{1}^{m-k}}{4}\left\|D^{2 k} v\right\|^{2}-\frac{\beta \varepsilon^{2}}{2}\left\|D^{m+k} u\right\|^{2}
$$

by assuming $(B)$ and Poincare inequality, we have

$$
\left(g\left(|u|^{2}\right) u,(-\Delta)^{k} v\right)=g\left(|u|^{2}\right) \frac{1}{2} \frac{d}{d t}\left\|D^{k} u\right\|^{2}+\varepsilon g\left(|u|^{2}\right)\left\|D^{k} u\right\|^{2} \geq \frac{1}{2} \frac{d}{d t}\left[g\left(|u|^{2}\right)\left\|D^{k} u\right\|^{2}\right]-\frac{C_{1} \lambda_{1}^{-m}}{2}\left\|D^{m+k} u\right\|^{2}+\varepsilon g\left(\left.u\right|^{2}\right)\left\|D^{k} u\right\|^{2}
$$

because $f \in H$, according to Holder inequality and Young inequality, can obtain

$$
\left(f(x),(-\Delta)^{k} v\right) \leq \frac{1}{\beta \lambda_{1}^{m-k}}\|f(x)\|^{2}+\frac{\beta \lambda_{1}^{m-k}}{4}\left\|D^{2 k} v\right\|^{2}
$$

substitute formula (5) - (9) into formula (4), we have

$$
\begin{gathered}
\frac{d}{d t}\left(\left\|D^{k} v\right\|^{2}+\mu\left\|D^{m+k} u\right\|^{2}+g\left(\left.u\right|^{2}\right)\left\|D^{k} u\right\|^{2}\right)+\left(\frac{\beta \lambda_{1}^{m}}{2}-2 \varepsilon-\varepsilon^{2}\right)\left\|D^{k} v\right\|^{2}+\left(2 \varepsilon \mu_{0}-\varepsilon^{2} \lambda_{1}^{-m}-\beta \varepsilon^{2}-C_{1} \lambda_{1}^{-m}\right)\left\|D^{m+k} u\right\|^{2} \\
+2 \varepsilon g\left(\left.u u\right|^{2}\right)\left\|D^{k} u\right\|^{2} \leq \frac{2}{\beta \lambda_{1}^{m-k}}\left\|D^{k} f\right\|^{2} \leq C
\end{gathered}
$$

where $2 \varepsilon \mu_{0}-\varepsilon^{2} \lambda_{1}^{-m}-\beta \varepsilon^{2}-C_{1} \lambda_{1}^{-m}>0$, and we can get

$$
\frac{d}{d t} \phi(t)+\gamma \phi(t) \leq C
$$

where $2 \varepsilon \mu_{0}-\varepsilon^{2} \lambda_{1}^{-m}-\beta \varepsilon^{2}-C_{1} \lambda_{1}^{-m}>0, \phi(t)=\left\|D^{k} v\right\|^{2}+\mu\left\|D^{m+k} u\right\|^{2}+g\left(\|\left. u\right|^{2}\right)\left\|D^{k} u\right\|^{2}$, then by Gronwall inequality, there are

$$
\phi(t) \leq \phi(0) e^{-\gamma t}+\frac{C}{\gamma}\left(1-e^{-\gamma}\right)
$$

Because $\mu>1, \min \{1, \mu\}=1$, so we can get

$$
\|(u, v)\|_{E_{k}}^{2}=\left\|D^{m+k} u\right\|^{2}+\left\|D^{k} v\right\|^{2} \leq \phi(0) e^{-\gamma t}+C\left(1-e^{-\gamma t}\right)
$$

where $k=0,1,2, \cdots, m$. Therefore, there exists a nonnegative real number $C\left(R_{1}\right)$ and $t=t_{2}(\Omega)>0$, such that

$$
\varlimsup_{t \rightarrow \infty}\|(u, v)\|_{E_{k}}^{2} \leq \frac{C}{\gamma}=C\left(R_{1}\right)\left(t>t_{2}\right)
$$


Lemma 1 is proved.

Theorem 1 (Existence and uniqueness of solutions) Under Lemma 1, then the initial boundary value problem (1) - (3) has a unique smooth solution $(u, v) \in L^{\infty}\left([0,+\infty) ; E_{k}\right)$.

Proof Let $u, v$ be the two solutions of equation (1), let $w=u-v$ be introduced into equation (1) and the inner product with $w_{t}$, can obtain

$$
\left(w_{t t}+M\left(\left\|D^{m} u\right\|^{2}\right)(-\Delta)^{m} u-M\left(\left\|D^{m} v\right\|^{2}\right)(-\Delta)^{m} v+\beta(-\Delta)^{m} w_{t}+g\left(\left.u\right|^{2}\right) u-g\left(\|\left. v\right|^{2}\right) v, \quad w_{t}\right)=0
$$

Formula (14) is dealt with item by item as follows:

$$
\begin{gathered}
\left(w_{t t}, w_{t}\right)=\frac{1}{2} \frac{d}{d t}\left\|w_{t}\right\|^{2} \\
\left(\beta(-\Delta)^{m} w_{t}, w_{t}\right)=\beta\left\|D^{m} w_{t}\right\|^{2}
\end{gathered}
$$

according to assume $(A)$ and differential mean value theorem, we can get

$$
\begin{gathered}
\left(M\left(\left\|D^{m} u\right\|^{2}\right)(-\Delta)^{m} u-M\left(\left\|D^{m} v\right\|^{2}\right)(-\Delta)^{m} v, w_{t}\right) \\
\geq \frac{1}{2} M\left(\left\|D^{m} u\right\|^{2}\right) \frac{d}{d t}\left\|D^{m} w\right\|^{2}-\left\|M^{\prime}(\xi)\right\|_{\infty}\left(\left\|D^{m} u\right\|+\left\|D^{m} v\right\|\left\|D^{m} w\right\|\left\|D^{m} v\right\|\left\|D^{m} w_{t}\right\| \geq \frac{\mu}{2} \frac{d}{d t}\left\|D^{m} w\right\|^{2}-\frac{C_{2}}{2}\left\|D^{m} w\right\|^{2}-\frac{C_{2}}{2}\left\|D^{m} w_{t}\right\|\right.
\end{gathered}
$$

by hypothesis $(B)$, Holder inequality, Poincare inequality and differential mean value theorem, we have

$$
\left(g\left(|u|^{2}\right) u-g\left(|v|^{2}\right) v, \quad w_{t}\right)=\left(g\left(\left.u\right|^{2}\right) w, \quad w_{t}\right)+\left(g^{\prime}(\xi)(|u|+|v|)|w| v, w_{t}\right) \geq-\left(\frac{C_{4} \varepsilon^{2}}{2}+\frac{C_{3}}{2}\right) \lambda_{1}^{-m}\left\|D^{m} w\right\|^{2}-\left(\frac{C_{4} \varepsilon^{2}}{2}+\frac{C_{3}}{2}\right)\left\|w_{t}\right\|^{2}
$$

substitute formula (15) - (18) into formula (14), we conclude that

$$
\frac{d}{d t}\left(\left\|w_{t}\right\|^{2}+\mu\left\|D^{m} w\right\|^{2}\right)+\left(2 \beta-C_{2}\right)\left\|D^{m} w_{t}\right\|^{2}-\left(\frac{C_{2}}{\mu}+\frac{C_{3}}{\mu} \lambda_{1}^{-m}+\frac{C_{4} \varepsilon^{2}}{\mu} \lambda_{1}^{-m}\right) \mu\left\|D^{m} w\right\|^{2}-\left(C_{3}+C_{4} \varepsilon^{2}\right)\left\|w_{t}\right\|^{2} \leq 0
$$

because when $\beta$ is large enough, if $2 \beta-C_{2} \geq 0$, then $\left(2 \beta-C_{2}\right)\left\|D^{m} w_{t}\right\|^{2}$ can be omitted temporarily, so the above formula can be written as

$$
\frac{d}{d t}\left(\left\|w_{t}\right\|^{2}+\mu\left\|D^{m} w\right\|^{2}\right) \leq\left(\frac{C_{2}}{\mu}+\frac{C_{3}}{\mu} \lambda_{1}^{-m}+\frac{C_{4} \varepsilon^{2}}{\mu} \lambda_{1}^{-m}\right) \mu\left\|D^{m} w\right\|^{2}+\left(C_{3}+C_{4} \varepsilon^{2}\right)\left\|w_{t}\right\|^{2}
$$

If we take $\sigma=\min \left\{\left(\frac{C_{2}}{\mu}+\frac{C_{3}}{\mu} \lambda_{1}^{-m}+\frac{C_{4} \varepsilon^{2}}{\mu} \lambda_{1}^{-m}\right), C_{3}+C_{4} \varepsilon^{2}\right\}$, then we have

$$
\frac{d}{d t} N(t)+\sigma N(t) \leq 0
$$

Where $N(t)=\left\|w_{t}\right\|^{2}+\mu\left\|D^{m} w\right\|^{2}$, by using the Gronwall inequality, we get

$$
N(t) \leq N(0) e^{-\sigma t}=0
$$

so $w=0$, the uniqueness is proved.

Theorem 2[9] Let $E$ be a Banach space, and the semi-group $S(t): E \rightarrow E$ satisfy the following conditions:

(1) The semi-group $S(t)$ is uniformly bounded in $E$, i. e. $\forall R>0$, and there exists a constant $C(R)$, so that when $\|u\|_{E} \leq R$, there is

$$
\|S(t) u\|_{E} \leq C(R) \quad(\forall t \in[0,+\infty))
$$

(2) There exists a bounded absorption set $B_{0}$ in $E$;

(3) $S(t) \quad(t \geq 0)$ is a fully continuous operator. Then semi-group $S(t)$ has compact global attractor $A_{0}$.

By replacing Banach space $E$ in Theorem 2 with Hilbert space $E_{k}$, the following theorem of global attractor family is obtained:

Theorem 3 Supposes that the global smooth solution of the 
problem (1) - (3) satisfies the condition of lemma 1, then the problem (1) - (3) has a global attractor family ${ }_{k}$, that is there (1) $S(t)_{A_{k}}=A_{k}, t>0$; exists a compact set $A_{k}$ such that

(2) $\lim _{t \rightarrow \infty} \operatorname{dist}\left(S(t) B, A_{k}\right)=0,\left(\forall B \subset E_{k}\right)$, where

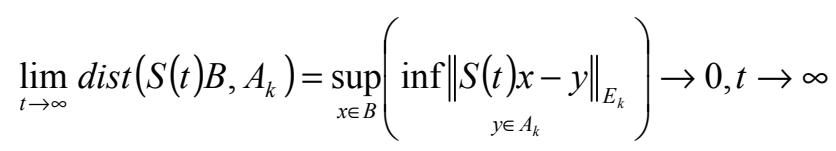

$S(t)$ is the solution semi-group generated by problem (1) - (3).

Proof It is necessary to verify the hypotheses of Theorem 2 (1), (2), (3). Under the hypothesis of Theorem 2, there exists a solution semi-group $S(t)$ :

$$
S(t): E_{k} \rightarrow E_{k}
$$

By Lemma 1, for $\forall B_{k} \subset E_{k}$ and contained in $\left\{\|u\|_{H_{0}^{m+k}(\Omega)}+\|v\|_{H_{0}^{k}(\Omega)}\right\} \leq R_{k}$ bounded set

$$
\left\|S(t)\left(u_{0}+v_{0}\right)\right\|_{E_{k}}^{2}=\|u\|_{H_{0}^{m+k}(\Omega)}^{2}+\|v\|_{H_{0}^{k}(\Omega)}^{2} \leq\left(\left\|u_{0}\right\|_{H_{0}^{m+k}(\Omega)}^{2}+\left\|v_{0}\right\|_{H_{0}^{k}(\Omega)}^{2}\right) \leq R_{k}^{2}
$$

where $t \geq 0,\left(u_{0}, v_{0}\right) \in B_{k}$, this shows that $\{S(t)\}(t \geq 0)$ is uniformly bounded in $E_{k}$. By Lemma 1 , there is further

$$
\left\|S(t)\left(u_{0}+v_{0}\right)\right\|_{E_{k}}^{2}=\|u\|_{H_{0}^{m+k}(\Omega)}^{2}+\|v\|_{H_{0}^{k}(\Omega)}^{2} \leq R_{k}^{2} \quad t \geq t_{0}=t_{0}\left(R_{k}\right)
$$

so $B_{0 k}$ is bounded absorption set of semi-group $S(t)$. Because $E_{k} \subset E_{0}$ is compact embedded, that is, bounded set in $E_{k}(k=1,2, \cdots, m)$ is compact set in $E_{0}$, so operator semi-group $S(t)$ is totally connected to $t \geq 0$, then the equation has a global attractor family $A_{k}=\omega\left(B_{0 k}\right)=\bigcap_{\tau \geq 0} \overline{\bigcup_{t \geq \tau} S(t) B_{0 k}}(k=1,2, \cdots, m)$.

\section{Dimension Estimation}

In this part, we first linearize the equation (1), then prove the Frechet differentiability of the solution semi-group, and finally prove the Haudorff dimension and Fractal dimension of the finite dimension of the global attractor family.

Linearization of equation (1) - (3) is obtained

$$
\begin{gathered}
U_{t t}+M\left(\left\|D^{m} u\right\|^{2}\right)(-\Delta)^{m} U+2 M^{\prime}\left(\left\|D^{m} u\right\|^{2}\right)\left(D^{m} u, D^{m} U\right)(-\Delta)^{m} u \\
+\beta(-\Delta)^{m} U_{t}+\left(g\left(\|\left. u\right|^{2}\right) 2 u^{2}+g\left(\|\left. u\right|^{2}\right)\right) U=0 \\
U(x, t)=0, x \in \partial \Omega, t>0 \\
U(x, 0)=\xi, U_{t}(x, 0)=\eta
\end{gathered}
$$

where $(\xi, \eta) \in E_{k},\left(u, u_{t}\right)=S(t)\left(u_{0}, u_{1}\right)$ is the solution of problem (1) - (3) obtained by $\left(u_{0}, u_{1}\right) \in A_{k}$.

Given $\left(u_{0}, u_{1}\right) \in A_{k}, S(t): E_{k} \rightarrow E_{k}$, we can prove that for any $(\xi, \eta) \in E_{k}$, the linear initial-boundary value problem (26) - (28) has a unique solution $\left(U(t), U_{t}(t)\right) \in L^{\infty}\left([0,+\infty) ; E_{k}\right)$.

Lemma 2 For any $t>0, R>0$, the mapping $S(t): E_{k} \rightarrow E_{k}$ is Frechet differentiable on $E_{k}$. The differential on $\vartheta=\left(u_{0}, u_{1}\right)^{T}$ is a linear operator on $F:(\xi, \eta)^{T} \rightarrow\left(U(t), U_{t}(t)\right)^{T}$, where $U(t), U_{t}(t)$ are solutions of the problem (26) - (28).

Proof Let $\varphi_{0}=\left(u_{0}, v_{0}\right)^{T} \in E_{k}, \widetilde{\varphi}_{0}=\left(u_{0}+\xi, v_{0}+\eta\right)^{T} \in E_{k}$, and $\left\|\varphi_{0}\right\|_{E_{k}} \leq R,\left\|\widetilde{\varphi}_{0}\right\|_{E_{k}} \leq R$, we define $(u, v)^{T}=S(t) \varphi_{0}$, $(\tilde{u}, \widetilde{v})^{T}=S(t) \widetilde{\varphi}_{0}$, can obtain Lipchitz property of bounded set $S(t)$ on $E_{k}$, i. e.

$$
\left\|S(t) \varphi_{0}-S(t) \widetilde{\varphi}_{0}\right\|_{E_{k}}^{2} \leq e^{c t}\|(\xi, \eta)\|_{E_{k}}^{2}
$$

let $\theta=\tilde{u}-u-U$ be the solution of equation

$$
\begin{gathered}
\theta_{t t}+M\left(\left\|D^{m} u\right\|^{2}\right)(-\Delta)^{m} \theta+\beta(-\Delta)^{m} \theta_{t}=h \\
\theta(0)=\theta_{t}(0)=0
\end{gathered}
$$

Let $s=\left\|D^{m} u\right\|^{2}, \widetilde{s}=\left\|D^{m} \tilde{u}\right\|^{2}$, then we can get

$$
\begin{array}{r}
h=(M(s)-M(\tilde{s}))(-\Delta)^{m} \tilde{u}+2 M^{\prime}(s)\left(D^{m} u, D^{m} U\right)(-\Delta)^{m} u \\
+g\left(|u|^{2}\right) u-g\left(|\widetilde{u}|^{2}\right) \tilde{u}+\left(g^{\prime}\left(|u|^{2}\right) 2 u^{2}+g\left(|u|^{2}\right)\right) U,(32)
\end{array}
$$

by taking the inner product of both sides of equation (30) and $\theta_{t}$, we get

$$
\frac{1}{2} \frac{d}{d t}\left\|\theta_{t}\right\|^{2}+M(s) \frac{1}{2} \frac{d}{d t}\left\|D^{m} \theta\right\|^{2}+\beta\left\|D^{m} \theta_{t}\right\|^{2}=\left(h, \theta_{t}\right),
$$

let $\bar{u}=u-\tilde{u}$, then by supposing $(A)$, adding one item twice, subtracting one item and generalized differential mean value 
theorem, we can obtain

$$
\begin{gathered}
\left((M(s)-M(\tilde{s}))(-\Delta)^{m} \tilde{u}+2 M^{\prime}(s)\left(D^{m} u, D^{m} U\right)(-\Delta)^{m} u, \theta_{t}\right) \\
=M^{\prime}(\alpha s+(1-\alpha) \tilde{s})\left(\left(D^{m} u, D^{m} u\right)-\left(D^{m} \tilde{u}, D^{m} \tilde{u}\right)\right)\left((-\Delta)^{m} \tilde{u}, \boldsymbol{\theta}_{t}\right)+2 M^{\prime}(s)\left(D^{m} u, D^{m} U\right)\left((-\Delta)^{m} u, \boldsymbol{\theta}_{t}\right) \\
\leq C_{5} M^{\prime \prime}(s)\left\|D^{m} \bar{u}\right\|^{2}\left\|D^{m} u+D^{m} \tilde{u}\right\|^{2}\left\|D^{m} \tilde{u}\right\| D^{m} \theta_{t}\left\|+M^{\prime}(s)\right\| D^{m} \bar{u}\left\|^{2}\right\| D^{m} u\left\|D^{m} \theta_{t}\right\| \\
+2 M^{\prime}(s)\left\|D^{m} \theta\right\| D^{m} u\|\| D^{m} \tilde{u}\left\|D^{m} \theta_{t}\right\|+2 M^{\prime}(s)\left\|D^{m}\right\|^{2}\left\|D^{m} u\right\| D^{m} \theta_{t}\|+\| D^{m} \theta\left\|D^{m} u\right\| D^{m} u\left\|D^{m} \theta_{t}\right\| \\
\leq C_{6}\left\|D^{m} \bar{u}\right\|^{2}\left\|D^{m} \theta_{t}\right\|+C_{7}\left\|D^{m} \bar{u}\right\|^{2}+\left\|D^{m} \theta\right\|\left\|D^{m} \theta_{t}\right\|+C_{8}\left\|D^{m} \bar{u}\right\|^{2}+\left\|D^{m} \theta\right\|\left\|D^{m} \theta_{t}\right\|
\end{gathered}
$$

according to hypothesis $(B)$, differential mean value theorem and Holder inequality, we can get

$$
\left(g\left(\left|u^{2}\right|^{2}\right) u-g\left(|\widetilde{u}|^{2}\right) \widetilde{u}+\left(g^{\prime}\left(|u|^{2}\right) 2 u^{2}+g\left(|u|^{2}\right)\right) U, \theta_{t}\right) \leq C_{9}\left\|g^{\prime \prime}(\widetilde{\omega})\right\|_{\infty}\|\bar{u}\|^{2}\left\|\theta_{t}\right\|+2\left\|g^{\prime}\left(\|\left. u\right|^{2}\right)^{2}\right\|_{\infty}\left\|u^{2}\right\| \theta \theta\left\|\theta_{t}\right\|+\| g\left(\left.u\right|^{2}\left\|_{\infty}\right\| \theta\|\| \theta_{t} \| \leq C_{10}\left(\|\bar{u}\|^{2}+\|\theta\|\left\|\theta_{t}\right\|\right.\right.
$$

so according to (34) - (35) formula and using Young's inequality, we have

$$
\left(h, \theta_{t}\right) \leq \frac{3 \varepsilon}{4}\left\|\theta_{t}\right\|^{2}+\left(\frac{\left(C_{7}+C_{8}\right)^{2}}{2 \beta}+\frac{C_{10}^{2}}{\varepsilon \lambda_{1}^{m}}\right)\left\|D^{m} \theta\right\|^{2}+\beta\left\|D^{m} \theta_{4}\right\|^{2}+C_{11}\left\|D^{m} \bar{u}\right\|^{4}
$$

Formula (36) is now substituted for formula (33), and according to hypothesis $(A)$, can obtain

$$
\frac{d}{d t}\left(\left\|\theta_{t}\right\|^{2}+\mu\left\|D^{m} \theta\right\|^{2}\right) \leq C_{12}\left(\left\|\theta_{t}\right\|^{2}+\mu\left\|D^{m} \theta\right\|^{2}\right)+C_{13}\left\|D^{m} \bar{u}\right\|^{4}
$$

Then by using Gronwall inequality, we conclude that

$$
\left\|\theta_{t}\right\|^{2}+\mu\left\|D^{m} \theta\right\|^{2} \leq C_{14} e^{C_{15} t} \int_{0}^{t}\left\|D^{m} \bar{u}\right\|^{4} d \tau \leq C_{16} e^{C_{17} t}\left\|(\xi, \eta)^{T}\right\|_{E_{k}}^{4}
$$

so from formula (38) we can get in $E_{k}$, let $B(t)=\left(U(t), U_{t}(t)\right)$, when $\left\|(\xi, \eta)^{T}\right\|^{2} \rightarrow 0$, we have

$$
\frac{\|\widetilde{\varphi}(t)-\varphi(t)-B(t)\|_{E_{k}}^{2}}{\left\|(\xi, \eta)^{T}\right\|_{E_{k}}^{2}} \leq C_{18} e^{C_{19} t}\left\|(\xi, \eta)^{T}\right\|_{E_{k}}^{2} \rightarrow 0
$$

Lemma 2 is proved.

Theorem 4 Under the condition of Theorem 3, the global attractor $A_{k}$ of the problem (1) - (3) has Hausdorff dimension and Fractal dimension, and $d_{H}\left(A_{k}\right)<\frac{1}{6} n, d_{F}\left(A_{k}\right)<\frac{7}{6} n$.

Proof Let $A=-\Delta$, equation (1) can be written as

$$
u_{t t}+M\left(\left\|A^{\frac{m}{2}} u\right\|^{2}\right) A^{m} u+\beta A^{m} u_{t}+g\left(|u|^{2}\right) u=f(x)
$$

let $\Psi=R_{\varepsilon} \phi=(u, v), \phi=\left(u, u_{t}\right), v=u_{t}+\varepsilon u, \quad R_{\varepsilon}:\left\{u, u_{t}\right\} \rightarrow\left\{u, u_{t}+\varepsilon u\right\}$ be an isomorphic mapping, so equation (40) can be written as

$$
\Psi_{t}+\Lambda_{\varepsilon} \Psi+\bar{g}(\Psi)=\bar{f}
$$


where $\Psi=\left\{u, u_{t}+\varepsilon u\right\}^{T}, \bar{g}(\Psi)=\left\{0, g\left(|u|^{2}\right) u\right\}, \bar{f}=\{0, f(x)\}^{T}, \Lambda_{\varepsilon}=\left(\begin{array}{cc}\|\left(\left\|\frac{m}{2}\right\|^{2}\right. & -I \\ \left.A^{2} u \|^{2}-\beta \varepsilon\right) A^{m}+\varepsilon^{2} I & \beta A^{m}-\varepsilon I\end{array}\right)$.

$$
\Psi_{t}:=F(\Psi)=\bar{f}-\Lambda_{\varepsilon} \Psi-\bar{g}(\Psi)
$$

Lemma 2 shows that $S(t): E_{k} \rightarrow E_{k}$ is a Frechet differential, then the linearized equation (42) can be written as

$$
P_{t}+\Lambda_{\varepsilon} P+\overline{g^{\prime}}(\Psi) P=0
$$

where $P_{t}=F_{t}(\Psi), P=\left(U, U_{t}+\varepsilon U\right), \bar{g}^{\prime}(\Psi)=\left(0,\left(g^{\prime}\left(\left.u\right|^{2}\right) \cdot 2 u^{2}+g\left(\left.u\right|^{2}\right)\right) U\right) . \quad U$ is the solution of equation (41).

For a fixed $\left(u_{0}, v_{0}\right) \in E_{k}$, let $\xi_{1}, \xi_{2}, \cdots, \xi_{n}$ be $n$ elements in $E_{k}$. Let $U_{1}(t), U_{2}(t), \cdots, U_{n}(t)$ be $n$ solutions of linear equation (43) with initial values $U_{1}(0)=\xi_{1}, U_{2}(0)=\xi_{2}, \cdots, U_{n}(0)=\xi_{n}$. By direct calculation, we can obtain

$$
\left\|U_{1}(t) \Lambda U_{2}(t) \Lambda \cdots \Lambda U_{n}(t)\right\|_{E_{k}}^{2}=\left\|\xi_{1} \Lambda \xi_{2} \Lambda \cdots \xi_{n}\right\|_{\Lambda E_{k}} \exp \left(\int_{0}^{t} t r F^{\prime}(\Psi(\tau)) \cdot Q_{n}(\tau) d \tau\right)
$$

where $\Lambda$ denotes the outer product, $\operatorname{tr}$ denotes the trace of the operator, and $Q_{n}(\tau)$ denotes the orthogonal projection of subspaces generated by $E_{k}$ to $U_{1}(t), U_{2}(t), \cdots, U_{n}(t)$.

For a given time $\tau$, let $\omega_{j}(\tau)=\left(\zeta_{j}(\tau), \eta_{j}(\tau)\right)^{T}, j=1,2, \cdots, n$ be the standard orthogonal basis of space $\operatorname{span}\left\{U_{1}(t), U_{2}(t), \cdots, U_{n}(t)\right\}$. Define the inner product on $E_{k}$

$$
((\zeta, \eta),(\bar{\zeta}, \bar{\eta}))=\left(\left(D^{m+k} \zeta, D^{m+k} \bar{\zeta}\right)+\left(D^{k} \eta, D^{k} \bar{\eta}\right)\right)
$$

To sum up, we can get

$$
\begin{aligned}
\operatorname{tr} F_{t}(\Psi(\tau)) \cdot Q_{n}(\tau) & =\sum_{j=1}^{n}\left(F_{t}(\Psi(\tau)) \cdot Q_{n}(\tau) \omega_{j}(\tau), \omega_{j}(\tau)\right)_{E_{k}} \\
& =\sum_{j=1}^{n}\left(F_{t}(\Psi(\tau)) \omega_{j}(\tau), \omega_{j}(\tau)\right)_{E_{k}}
\end{aligned}
$$

where $\left(F_{t}(\Psi(\tau)) \omega_{j}(\tau), \omega_{j}(\tau)\right)_{E_{k}}=-\left(\Lambda_{\varepsilon} \omega_{j}, \omega_{j}\right)-\left(\overline{g^{\prime}}(\Psi) \omega_{j}, \omega_{j}\right)$. From Poincare inequality and hypothesis $(A)$, we can obtain

$$
\begin{gathered}
-\left(\Lambda_{\varepsilon} \omega_{j}, \omega_{j}\right)=-\left(\left(\varepsilon \zeta_{j}-\eta_{j},\left(M\left(\left\|A^{\frac{m}{2}} u\right\|^{2}\right)-\beta \varepsilon\right) A^{m} \zeta_{j}+\varepsilon^{2} \zeta_{j}+\beta A^{m} \eta_{j}-\varepsilon \eta_{j}\right),\left(\zeta_{j}, \eta_{j}\right)\right) \\
\leq-\varepsilon\left\|D^{m+k} \zeta_{j}\right\|^{2}+\frac{\beta \varepsilon+1-M\left(\left.\left\|A^{\frac{m}{2} u}\right\|\right|^{2}\right)}{2}\left(\left\|D^{m+k} \zeta_{j}\right\|^{2}+\left\|D^{m+k} \eta_{j}\right\|^{2}\right)+\frac{\varepsilon^{2}}{2}\left(\left\|D^{k} \zeta_{j}\right\|^{2}+\left\|D^{k} \eta_{j}\right\|^{2}\right)-\beta\left\|D^{m+k} \eta_{j}\right\|^{2}+\varepsilon\left\|D^{k} \eta_{j}\right\|^{2} \\
\leq \frac{\beta \varepsilon+\varepsilon^{2} \lambda_{1}^{-m}+1-M\left(\| A^{\frac{m}{2} u \|^{2}}\right)-2 \varepsilon}{2}\left\|D^{m+k} \zeta_{j}\right\|^{2}-\frac{\left(2 \beta+M\left(\left\|A^{\frac{m}{2}} u\right\|^{2}\right)-\beta \varepsilon-1\right) \lambda_{1}^{m}}{2}\left\|D^{k} \eta_{j}\right\|^{2}+\frac{\varepsilon^{2}+2 \varepsilon}{2}\left\|D^{k} \eta_{j}\right\|^{2} \\
\leq-\frac{C_{20}}{2}\left(\left\|D^{m+k} \zeta_{j}\right\|^{2}+\left\|D^{k} \eta_{j}\right\|^{2}\right) \\
\text { where } \frac{M\left(\left\|A^{\frac{m}{2}} u\right\|^{2}\right)-1}{\beta}<\varepsilon<\frac{\sqrt{(\beta-2)^{2}+4 \lambda_{1}^{-m}\left(M\left(\left\|A^{\frac{m}{2}} u\right\|^{2}\right)-1\right)}-(\beta-2)}{2 \lambda_{1}^{-m}}
\end{gathered}
$$




$$
C_{20}=\min \left\{-\left(\beta \varepsilon+\varepsilon^{2} \lambda_{1}^{-m}+1-M\left(\left\|A^{\frac{m}{2}} u\right\|^{2}\right)-2 \varepsilon\right),\left(2 \beta+M\left(\left\|A^{\frac{m}{2}} u\right\|^{2}\right)-\beta \varepsilon-1\right) \lambda_{1}^{m}-\varepsilon^{2}-2 \varepsilon\right\} .
$$

According to the definition of inner product over $E_{k}$, Holder inequality and hypothesis $(B)$, we can get

$$
\begin{aligned}
\left.\mid \overline{g^{\prime}}(\Psi) \omega_{j}, \omega_{j}\right) \mid & \left.=\left(\| 0,\left(g^{\prime}\left(|u|^{2}\right) \cdot 2 u^{2}+g\left(|u|^{2}\right)\right) \eta_{j}\right),\left(\zeta_{j}, \eta_{j}\right)\right) \\
& =\left(D^{k}\left(g^{\prime}\left(|u|^{2}\right) \cdot 2 u^{2}+g\left(|u|^{2}\right)\right) \eta_{j}, D^{k} \eta_{j}\right) \\
& \geq-\left(\left\|g^{\prime}\left(|u|^{2}\right)\right\|_{\infty}\left\|2 u^{2}\right\|+\left\|g\left(\|\left. u\right|^{2}\right)\right\|_{\infty}\right)\left\|D^{k} \eta_{j}\right\|^{2} \\
& \geq-r\left\|D^{k} \eta_{j}\right\|^{2}
\end{aligned}
$$

According (46),(47) and (48), we have

$$
\left(F_{t}(\Psi(\tau)) \omega_{j}(\tau), \omega_{j}(\tau)\right)_{E_{k}} \leq-\frac{C_{20}}{2}\left(\left\|D^{m+k} \zeta_{j}\right\|^{2}+\left\|D^{k} \eta_{j}\right\|^{2}\right)+r\left\|D^{k} \eta_{j}\right\|^{2} \leq-\frac{C_{21}}{2}\left(\left\|\zeta_{j}\right\|^{2}+\left\|\eta_{j}\right\|^{2}\right)+r\left\|D^{k} \eta_{j}\right\|^{2}
$$

Because $\omega_{j}(\tau)=\left(\zeta_{j}(\tau), \eta_{j}(\tau)\right)^{T}, j=1,2, \cdots, n$ are the standard orthogonal bases of space $\operatorname{span}\left\{U_{1}(t), U_{2}(t), \cdots, U_{n}(t)\right\}$, so

$$
\left\|\zeta_{j}\right\|^{2}+\left\|\eta_{j}\right\|^{2}=1
$$

then have

$$
\sum_{j=1}^{n}\left(F_{t}(\Psi(\tau)) \omega_{j}(\tau), \omega_{j}(\tau)\right)_{E_{k}} \leq-\frac{n C_{21}}{2}+r \sum_{j=1}^{n}\left\|D^{k} \eta_{j}\right\|^{2}
$$

For almost all $t$, there is $\sum_{j=1}^{n}\left\|D^{k} \eta_{j}\right\|^{2} \leq \sum_{j=1}^{n} \lambda_{j}^{s-1}$, so there have

$$
\begin{array}{r}
t r F_{t}(\Psi(\tau)) \cdot Q_{n}(\tau) \leq-\frac{n C_{21}}{2}+r \sum_{j=1}^{n} \lambda_{j}^{s-1} \\
\text { Let } q_{n}(t)=\sup _{\substack{\Psi_{0} \in A \\
\eta_{j} \in E \\
\left\|\eta_{j}\right\| \leq 1}}\left(\frac{1}{t} \int_{0}^{t} t r F_{t}\left(S(\tau) \Psi_{0}\right) \cdot Q_{n}(\tau) d \tau\right)
\end{array}
$$

and have

$$
q_{n}=\lim _{t \rightarrow \infty} \sup q_{n}(t)
$$

then from formulas (53) and (54), we can see that

$$
q_{n} \leq-\frac{n C_{21}}{2}+r \sum_{j=1}^{n} \lambda_{j}^{s-1}
$$

Therefore, the Lyapunov exponent of $A_{k}$ is uniformly bounded

$$
\sigma_{1}+\sigma_{2}+\cdots+\sigma_{n} \leq-\frac{n C_{21}}{2}+r \sum_{j=1}^{n} \lambda_{j}^{s-1}
$$

then there exists a $s \in[0,1]$, such that

$$
\left(q_{j}\right)_{+} \leq-\frac{n C_{21}}{2}+r \sum_{j=1}^{n} \lambda_{j}^{s-1} \leq r \sum_{j=1}^{n} \lambda_{j}^{s-1} \leq \frac{n C_{21}}{7}
$$

where $\lambda_{j}$ are the eigenvalues of $A_{k}^{m}$, and $\lambda_{1}<\lambda_{2}<\cdots<\lambda_{m}$, then there have

$$
q_{n} \leq-\frac{{ }^{n} C_{21}}{2}\left(1-\frac{2 r}{n} C_{21} \sum_{j=1}^{n} \lambda_{j}^{s-1}\right) \leq-\frac{5}{14} n C_{21}
$$

so

$$
\max _{1 \leq j \leq n} \frac{\left(q_{j}\right)_{+}}{\left|q_{n}\right|} \leq \frac{1}{6}
$$

Then we can get conclusion $d_{H}(A)<\frac{1}{6} n, d_{F}(A)<\frac{7}{6} n$. The proof is complete.

\section{Conclusions}

On the basis of previous studies on global attractors, this paper studies the global attractor family on space $E_{k}(k=1,2, \cdots, m)$, and further studies the dimension estimation of global attractor family. However, the proof of the global attractor family in this paper is not sufficient, so the proof of the global attractor family needs to be further improved. It is suggested to further study and expand the global attractor family on this basis.

\section{Acknowledgements}

We express our sincere thanks to the anonymous reviewer for his/her careful reading of the paper, we hope that we can get valuable comments and suggestions. Making the paper better. 


\section{References}

[1] Kirchhoff G. Vorlesungen uber Mechanik [M]. Teubner: Sluttgart, 1883.

[2] Kosuke Ono. On global existence, asymptotic stability and blowing up of solutions for some degenerate non-linear wave equations of Kirchhoff type with strong dissipation [J]. Mathematical Methods in the Applied Sciences, 1997, 20(2):151-177.

[3] Xiaoming Fan, Yaguang Wang. Fractal dimension of attractors for a stochastic wave equation with nonlinear damping and white noise [J]. Stochastic Analysis and Applications, 2007, 25(2):381-396.

[4] Zhijian Yang, Yunqing Wang. Global attractor for the Kirchhoff type equation with a strong dissipation [J]. J. Differential Equations. 2010, 249: 3258-3278.

[5] Yang Zhijian, Jianling Cheng. Asymptotic Behavior of Solutions of Kirchhoff Equations [J]. Journal of Mathematical Physics. 2011, 31A (4): 1008-1021.

[6] M. M. Cavalcanti. Existence and exponential decay for a Kirchhoff-Carrier model with viscosity [J]. Journal of Mathematical Analysis and Applications, 1998, 226:40-60.

[7] Mitsuhiro Nakao. Global attractors for nonlinear wave equations with nonlinear dissipative terms [J]. Journal of Differential Equations, 2006, 227(1):204-229.

[8] Varga Kalantarov, Sergey Zelik. Finite-dimensional attractors for the quasi-linear strongly-damped wave equation [J]. Journal of Differential Equations, 2009, 247:1120-1155.

[9] Guoguang Lin. Nonlinear Evolution Equation [M]. Kunming: Yunnan University Press, 2011:16-123.

[10] Zhengde Dai. Dai Zhengde Papers Collection [C]. Kunming: Yunnan University Press, 2016.

[11] Penghui Lv, Jingxin Lu and Guoguang Lin. Global attractor for a class of nonlinear generalized Kirchhoff models [J]. Journal of Advances in Mathematics, 2016, 12(08): 6452-6462.
[12] Zhijian Yang, Xiao Li. Finite dimensional attractors for the Kirchhoff equation with a strong dissipation [J]. Journal of Mathematical Analysis and Applications, 2011, 375(2): 579-593.

[13] Igor Chueshov. Longtime dynamics of Kirchhoff wave models with strong nonlinear damping [J]. Journal of Differential Equations, 2011, 252(2): 1229-1262.

[14] Zhijian Yang, Pengyan Ding. Longtime dynamics of the Kirchhoff equation with strong damping and critical nonlinearity on RN [J]. Journal of Mathematical Analysis and Applications, 2016, 434(2): 1826-1851.

[15] Zhijian Yang, Pengyan Ding, Zhiming Liu. Global attractor for the Kirchhoff type equations with strong nonlinear damping and supercritical nonlinearity [J]. Applied Mathematics letters. 2014, 33(1): 12-17.

[16] Yuting Sun, Yunlong Gao, Guoguang Lin. The global attractor for the higher-order Kirchhoff-type equation with nonlinear strongly damped term [J]. International Journal of Modern Nonlinear Theory and Application. 2016, 5: 203-217.

[17] Ling Chen, Wei Wang, Guoguang Lin. The global attractor and their Hausdorff and Fractal dimensions estimation for the higher-order nonlinear Kirchhoff-type equation [J]. International Journal of Advances in Mathematics. 2016, 12(09): 6608-6621.

[18] Penghui Lv, Jinxin Lu, Guoguang Lin. Global attractor a class of nonlinear generalized Kirchhoff models [J]. Journal of Advances in Mathematics. 2016, 12(08): 6452-6462.

[19] Guoguang Lin, Sanmei Yang. Hausdorff dimension and Fractal dimension of the global attractor for the higher-order coupled Kirchhoff-type equations [J]. Journal of Applied Mathematics and Physics. 2017, 5: 2411-2424.

[20] Penghui Lv. Long-time behavior of a class of generalized nonlinear Kirchhoff-Boussinesq equations [D]. Kunming: Yunnan University, 2016. 